


\section{Imaging: ultrasound in lung disease}

\section{Educational aims}

1 To outline the situations where US is indicated.

1 To discuss the importance of EBUS in the diagnosis and staging of lung cancer.

I To encourage the pulmonologist to learn ultrasonic techniques, particularly transthoracic US, and emphasise the importance of formal training programmes.

\section{Summary}

Ultrasound (US) has received increased interest from chest physicians in recent years. Modern US devices are user friendly, inexpensive, lightweight and portable, which makes them suitable for outpatient settings, as well as for bedside investigations of the severely ill.

However, the view during bronchoscopy is limited to the inner surface in the case of parabronchial lesions. Therefore, endobronchial ultrasound (EBUS) systems were developed. In trials, it has been shown that, with the help of EBUS, the diagnosis and staging of lung cancer and other pathologies can be improved. EBUS is a safe technique and, so far, has proved extremely useful during diagnostic and interventional procedures. US is set to become a practical and essential tool for the pulmonologist in the near future.

This review aims to assess the most important and interesting articles in the field and to encourage the pulmonologist to learn ultrasonic techniques, particularly with regard to transthoracic US.

\section{Transthoracic ultrasound}

\section{Equipment}

Chest US can be performed with any modern US unit. A 5-7.5-MHz curvilinear probe allows visualisation of deeper structures, and the sector scan field allows a wider field of view through a small acoustic window. The chest wall, pleura and lungs may be quickly surveyed with the curvilinear probe. Once an abnormality has been identified, a high-resolution 7.5-10-MHz linear probe can be used to provide a detailed depiction. Both grey-scale and colour Doppler imaging are useful for the assessment of pleural and parenchymal abnormalities $[1,2]$.

\section{Technique}

Before performing US examination, it is important to review a patient's chest radiography to localise the area of interest. Maximum visualisation of the lungs and pleural space can be achieved by scanning along the intercostal spaces. Scanning should be performed during quiet respiration [3].
The ERS designates this

educational activity for a

maximum of one CME credit.

For information on how to

earn CME credits, see page

200.

\section{F.J.F. Herth \\ R. Eberhardt}

Dept of Pneumology and Critical Care Medicine, Thoraxklinik University of Heidelberg, Heidelberg, Germany.

\section{Correspondence:}

F.J.F. Herth

Dept of Pneumology and Critical Care Medicine

Thoraxklinik University of

Heidelberg

Amalienstraße 5

D-69126 Heidelberg

Germany

Fax: 496221396602

E-mail: Felix.Herth@thoraxklinikheidelberg.de

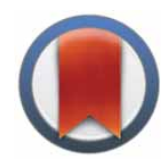

CrossMark $\leftarrow$ click for updates 
Initially, in the localised patient, investigation should be conducted via the subcostal and intercostal views. For the right pleural space, the liver serves as an acoustic window and the spleen for the left pleural space. During investigation in the subcostal view, the cutting plane is arranged far after cranial. Pathological processes in the upper pleural area can be examined using the upper thoracic aperture. On grey-scale images, the echogenicity of a lesion can be compared with that of the liver and characterised as hypoechoic, isoechoic or hyperechoic. In addition, the transducer is presented in the fossa supraclavicularis. Arteria and vena subclavia serve as guidance structures [2].

Sonographic views of the upper anterior and middle mediastinum can be obtained using the suprasternal approach. The suprasternal approach allows adequate assessment of the upper mediastinum in $90-95 \%$ of cases. This is performed with the patient in a supine position, with shoulders supported by a pillow and the head extended backward. Colour Doppler US is helpful in distinguishing the great vessels from any mediastinal mass [1,2].

With the previously mentioned equipment, the normal chest wall appears as a series of echogenic soft-tissue layers, representing the layers of muscle and the fascia planes. Below the soft tissue of the chest wall, the ribs appear as curvilinear structures on transverse scans, associated with posterior acoustic shadowing (figure 1). When the ribs are scanned along the long axis, the anterior cortex should appear as a continuous smooth echogenic line $[1,2]$. Beyond the pleuralung interface, the lung is air filled, and does not allow further visualisation of normal lung parenchyma [2]. The diaphragm is best examined through the lower intercostal spaces, and is seen as an echogenic line (1-mm thick) above the liver and spleen. Normal downward movement of the diaphragm should be seen on inspiration.

\section{Figure 1}

Normal US appearance of the chest. Below the relatively echogenic subcutaneous tissue, the intercostal muscles appear hypoechoic, but contain multiple echogenic fascia planes. The pleural interface appears as an echogenic line (IC). The arrow shows a rib.

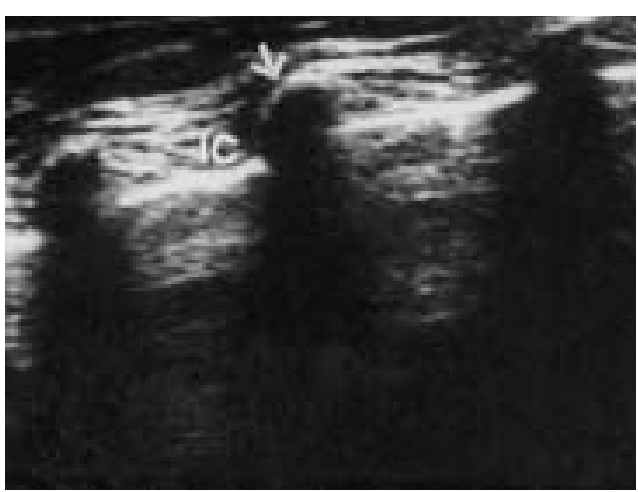

\section{Indications}

\section{Pleural effusions}

Pleural effusions with a volume of $\geq 30 \mathrm{~mL}$ can be recognised with US. The classical appearance of a pleural effusion is an echo-free layer between the visceral and parietal portions of the pleura (figure 2). The shape of the pleural effusion may vary with respiration and posture. In inflammatory effusions, lung sliding may be absent above the effusion, as a result of lung adhesion between the visceral and parietal portions of the pleura. Fibrinoid membranes within the effusion can be used to identify and highlight pulse-synchronous and breath-dependent mobility [3].

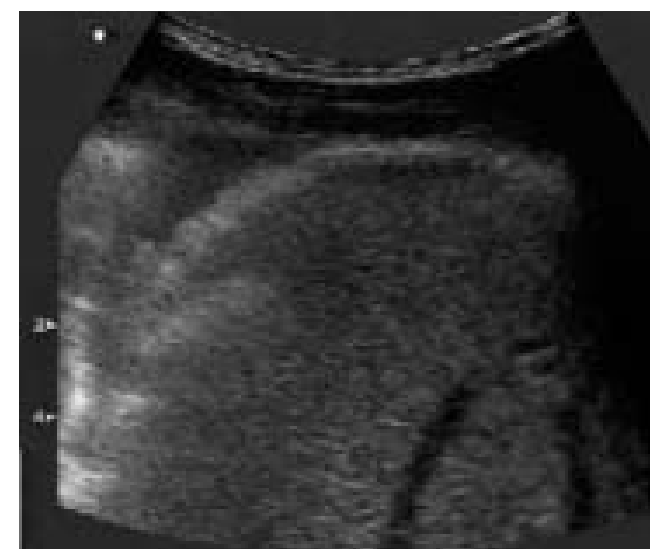

Figure 2

An anechoic effusion with passive atelectasis of the underlying lung.

The sonographic appearance of a pleural effusion depends on the cause, nature and chronicity of the collection. Echogenic, anechoic, complex but non-septated, and complex and septated appearances can be identified [4]. Transudates are almost invariably anechoic. However, exudates may appear anechoic, complex or echogenic. Effusions that are complex, septated or echogenic are usually exudates. Mobile strands of echogenic tissue and septations are frequently observed in inflammatory effusions $[2,5]$.

The ultrasonographic characteristics of a lung abscess include the following: an irregular wall width, a blurred outer margin, an oval or round shape, an acute chest wall angle and a negative pleural separation. The ultrasonographic characteristics of empyema comprise the following: a uniform wall width, a sharp outer margin, a lenticular shape, an obtuse chest wall angle and a positive pleural separation.

In a prospective study, Wu et al. [5] concluded that chest ultrasonography is a useful tool in the differentiation between lung abscesses and 
empyemas, and that ultrasonography alone is sufficient to make a correct diagnosis in most cases.

YU et al. [6] compared US with computed tomography (CT) in the assessment of 50 patients with unilateral opaque hemithorax. Forty-one of these patients had pleural effusions, with or without other abnormalities. US had a sensitivity of $95.1 \%$ for pleural lesions and $82.8 \%$ for parenchymal lesions. Not surprisingly, the depiction of mediastinal abnormality was poorer with US than with CT. However, in six patients, US showed pleural and parenchymal disease that was not identified with CT.

\section{Pleural thickening}

Pleural thickenings present themselves as constant echo-poor regions. Their demarcation, in contrast to effusions, mesothelioma and pleura near lung tumours, is difficult. A breath-dependent change in configuration is explained by effusion, a regular delimitation by pleural thickening and an irregular change by tumour [7]. Abscesses are fixed with the environment and, therefore, are not breath dependent.

Previous asbestos exposure is a relatively common cause of pleural thickening and may be confirmed if calcified pleural plaques are evident. These plaques cause focal areas of dense reflectivity with dense posterior acoustic shadowing, often with evidence of adjacent non-calcified pleural thickening.

\section{Pleural masses}

Pleural masses present themselves sonographically as irregularly limited, echo-poor, knotty or planar widenings along the pleura. As the thickness increases, so too does the probability of a malignant process. A widening of the pleura of $>1 \mathrm{~cm}$ results in a high-grade suspicion of a malignant tumour.

Usually, an accompanying pleural effusion, which is characteristically seen over large areas, additionally expands and is locally very pronounced. An effusion is helpful for the identification of a tumour and favours the sonographic differentiation of the pleura parietalis and visceralis.

The breath dependence of the lungs during the breath cycle is reduced when there is a pleural mass. Particularly pronounced echo-rich and irregular reflexes are found at the transition of the ventilated lung, and they correspond to reinforcement artefacts [8]. Sometimes pneumonias and atelectasis, existing at the same time, can be determined. Occasionally, in the advanced stage of the illness, a tumour infiltration can also be recognised in the lung tissue, standing out as echo-poor oblong structures against the ventilated lung. In addition, a locally progressing growth along puncture channels and by the diaphragm can be represented as an echo-poor [4].

Metastases may also appear as diffuse thickening of the parietal pleura and, to a lesser extent, the visceral pleura. Malignant pleural disease may invade the chest wall, with poor demarcation of the pleural mass and infiltration into the chest wall $[1,2]$.

\section{Pneumothorax}

Although a pneumothorax can usually be seen on chest radiography, a small pneumothorax may be overlooked on a radiograph of a supine patient obtained in the intensive care unit (ICU). The absence of lung sliding, exaggerated horizontal artefacts, loss of comet-tail artefacts and broadening of the pleural line in a band are the key sonographic signs $[9,10]$.

Bedside sonography is useful for excluding pneumothorax. Use of a combination of absent lung sliding and the loss of comet-tail artefacts has a reported sensitivity of $100 \%$, specificity of $96.5 \%$, and negative predictive value of $100 \%$ [9]

\section{Pulmonary embolism}

Using US, an area of pulmonary infarction may be recognised as a peripheral wedge-shaped hypoechoic region [11]. Early infarcts are less well defined, becoming more demarcated with time. A central hyperechoic structure, corresponding to a bronchiole, may be visualised (figure 3). In addition, a congested vessel leading into the infarct may be seen. As in sonography conducted for pneumonia, the area of pulmonary infarct demonstrable with US is usually smaller than that seen with angiography or scintigraphy.

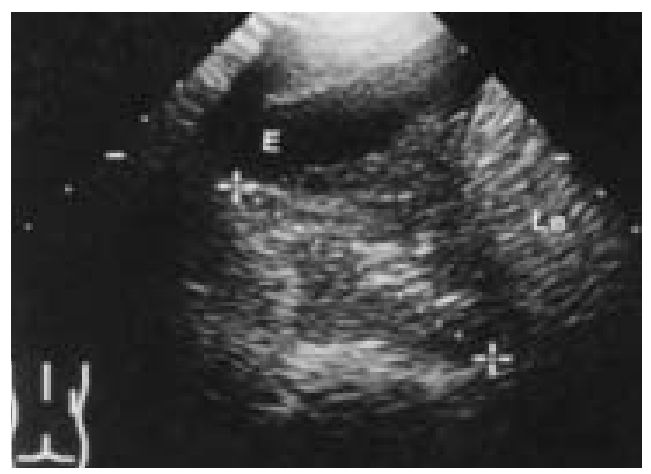

Figure 3

Early sonographic findings in pulmonary infarction. E: small pleural effusion; Le: liver. 


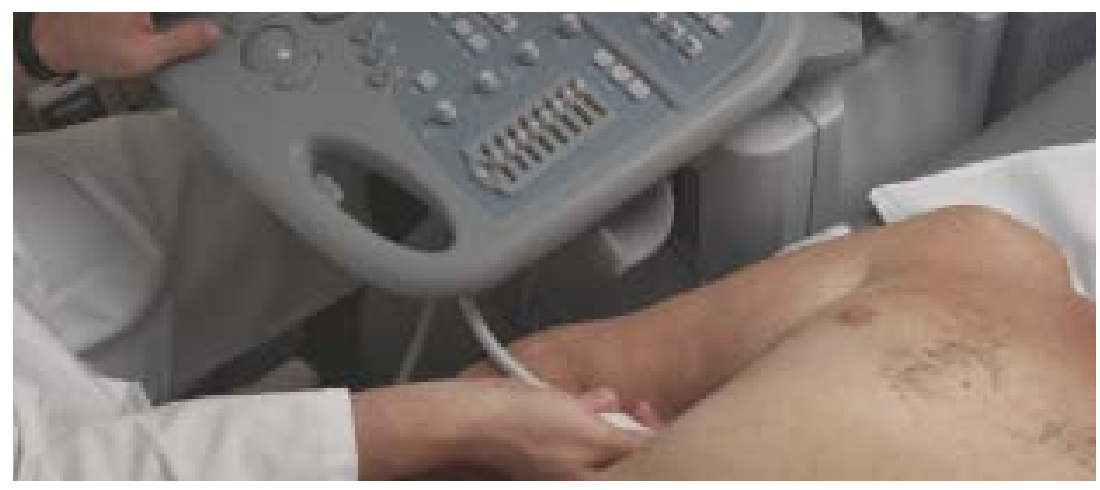

Although this technique has been advocated with enthusiasm, with a reported sensitivity of $77-98 \%$ and specificity of $66-83 \%$ [11], this experience has not been universal. With the increasing use of pulmonary CT angiography, sonography is unlikely to change current clinical practice in the initial diagnosis of pulmonary embolism.

\section{Puncture}

Benign pleural tumours present themselves as echo-poor, smoothly and sharply limited, roundish forms. They have a homogeneous echo texture and a relatively broad, as well as echo-rich, delimitation. A local infiltrative growth into the lung tissue or the chest wall is not seen. Pleural thickenings are usually echo rich and smoothly limited, as well as homogeneous. Pleural metastases present themselves as echo-poor to moderately echo-close, roundish, small knotty and laminar forms with irregular delimitation. Contrary to pleural metastasis, mesothelioma more frequently reveal laminar propagation.

There is no diagnostic criterion that can be used to differentiate pleural masses from pleural tumours (e.g. carcinomatosis of pleura, non-specific pleuritis). Therefore, histological proof of the diagnosis is essential $[1,2,4]$. Therefore, puncture can be carried out, either by means of fine, handguided or automated puncture needle. The area to be studied must be identified and definitively localised before the puncture.

The most important contraindications for this diagnostic method are considered to be a lack of cooperation, heavy coagulating disturbances and an advanced lung illness with a forced expiratory volume in one second $<1.0 \mathrm{~L}$. Furthermore, every patient has to sign a written consent form accepting the risks.

US can be used to guide biopsy of the pleura, either with a standard Tru-cut needle or an automated cutting needle device (18- or 20-gauge). Biopsy may be performed by using either a needle guide with the US probe or by means of freehand needle insertion. The passage of the tip of the needle can be seen in real time, confirming sampling of the lesion (figure 4). US has a reported sensitivity of $97 \%$ and accuracy of $98 \%$ for targeted diagnosis of peripheral lung cancer [12]. In tumours exhibiting central necrosis, US is particularly helpful in directing the biopsy procedure to the solid viable portions of the tumour, with improved sensitivity [13]. Biopsy with a cutting needle is preferable to fine-needle aspiration (FNA), due to its higher diagnostic yield, and, because it allows identification of histological subtypes, it has a higher specificity for benign lesions.

Complications in the context of punctures include: bleeding, infections, violation of the pleura visceralis with pneumothorax, haemoptysis, tumour cell kidnapping from the tumour in the container system, as well as local tumour cell propagation along the transthoracic puncture line $[1,2,4]$.

Even if the frequency of bleedings and lung violations can be reduced by means of US, complications are not completely eradicated. The complication rate for puncture of peripheral lesions by means of US or CT is comparable and amounts to $\sim 2-3 \%$ with experienced examiners. Overall, US-guided percutaneous lung biopsy is extremely safe, with an overall complication rate of $\sim-4 \%[1,2,4]$. Pneumothorax and haemoptysis are the most commonly encountered complications. However, in most cases, no treatment is needed. US, in common with other imaging modalities, may show the development of a pneumothorax at the time of biopsy.

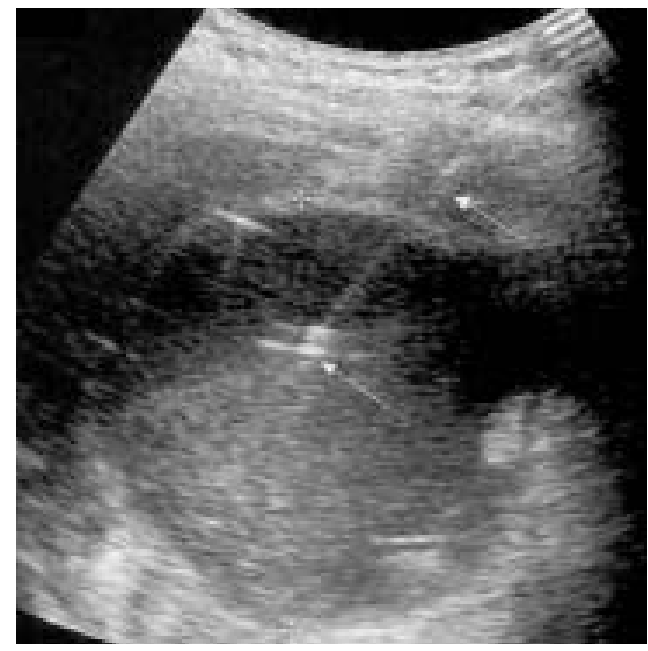

Figure 4

Needle puncture of a pleural mass. The needle tip is seen in the lesion (as indicated by arrows). 


\section{Endobronchial ultrasound}

The endoscopist's view through a bronchoscope is limited to the lumen and the internal surface of the airways. Processes within the airway wall and outside the airways can only be assessed by indirectsigns. Therefore, expanding the endoscopist's view beyond the airways is essential. The use of endoscopic US is promising in this regard and has already been proven to be valuable in other endoscopic specialities, such as gastro-intestinal applications.

Two EBUS techniques are available on the market: the so-called miniprobe and the transbronchial needle aspiration-endobronchial ultrasound (TBNA-EBUS) scope.

For applications inside the central airways, balloon-tipped flexible catheters and probes have been developed, which allow circular contact for the US waves (also known as "miniprobes"). These probes provide $a 360^{\circ}$ image of the airway wall, as well as the parabronchial and paratracheal structures. As the balloon enhances the US, penetration of the waves produced by $20-\mathrm{MHz}$ probes is increased. Thus, under favourable conditions, structures at a distance of up to $4 \mathrm{~cm}$ can be visualised. The probes can be applied with regular flexible endoscopes that have a biopsy channel diameter of $\geq 2.6 \mathrm{~mm}[14,15]$.

Recently, a bronchoscope with an integrated curvilinear electronic transducer at the tip (BFUC40P; Olympus Medical Co., Tokyo, Japan) has been developed that makes needle punctures under real-time endoscopic control possible. The endoscope has a biopsy channel diameter of 2 $\mathrm{mm}$. The ultrasonic frequency is $7.5 \mathrm{MHz}$, with a penetration depth of $5 \mathrm{~cm}$. The scanning direction is parallel to the longitudinal axis of the endoscope with a scanning angle of $50^{\circ}$, which enables full ultrasonic monitoring of a needle inserted through the biopsy channel during scanning [16].

\section{Indications}

For some indications, the superiority of US over conventional imaging has been proven in prospective studies, and, in many centres, EBUS is already established as a routine procedure. Current indications, according to the structures that can be analysed, are the evaluation of endoluminal, intramural and parabronchial structures; medical indications are early detection and tumour staging, inflammatory destruction of the airways, mediastinal lesions and malformations of mediastinal structures.

\section{Early cancer}

In small, radiologically invisible tumours, the decision to use local endoscopic therapeutic intervention depends on the intraluminal and intramural extent of the tumour within the different layers of the bronchial wall. In contrast to radiological imaging, EBUS allows even very small tumours, measuring only a few millimetres, to be analysed. As KURIMOTo et al. [17] demonstrated, EBUS is a very reliable tool for analysing the extent of these small lesions (figure 5). It has been demonstrated that the use of EBUS assessment in small autofluorescence (AF)-positive lesions, which were negative in white-light bronchoscopy, improved specificity (i.e. predicting malignancy), rising from 50 to $90 \%[18,19]$. Combining EBUS with AF has been proven to be efficient in prospective studies and has become the basis for curative endobronchial treatment of malignancies in some institutions.

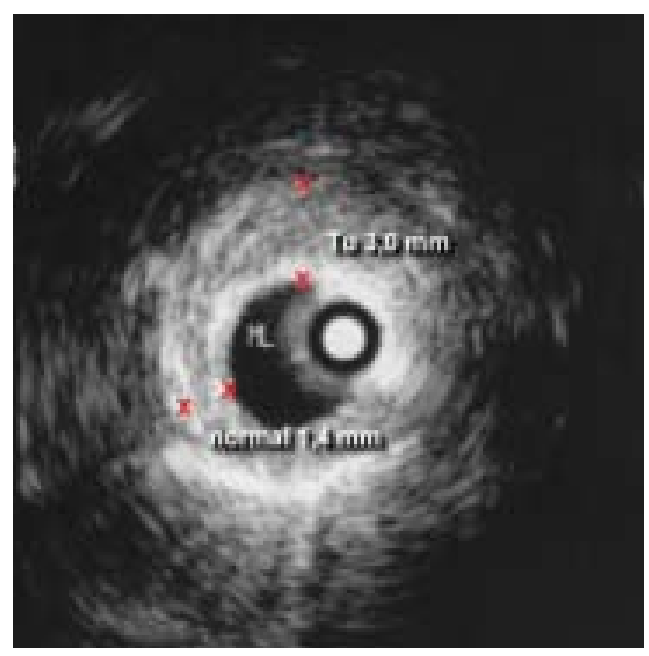

The most important article on this indication is by MirAZU et al. [19], who used EBUS findings to make therapy decisions in patients with early cancer lesions. In a study of 18 patients, nine patients were identified with tumours limited to the bronchial wall; these nine patients were treated with photodynamic therapeutic. The other nine patients had extracartilaginous tumours unsuspected by $\mathrm{CT}$ imaging, and were treated non-endoscopically (i.e. with surgery, radiation and chemotherapy). Using the EBUS findings as a decision maker, MiYAZu et al. [19] achieved a $100 \%$ complete remission rate in the endoluminal-treated group. Ata mean follow-up time of 32 months, none of the patients had experienced a recurrence. Earlier published trials using endoluminal techniques in early cancer lesions without
Figure 5

Early cancer lesion in the middle lobe (ML). The slightly enlarged bronchial wall (Tu) is seen compared to the normal wall (normal). 
EBUS assessment showed a significant failure and recurrence rate. Apparently, adding EBUS to the airway assessment in patients with presumed carcinoma in situ significantly increases the likelihood of identifying the patients best treated by endoluminal therapy.

\section{Advanced cancer}

In pre-operative staging, EBUS allows detailed analysis of intraluminal, submucosal and intramural tumour spread, which can be essential for decisions regarding resection margins. EBUS is especially useful in diagnosing mediastinal tumours involving the large vessels (e.g. the aorta, the cava and the main pulmonary arteries) and the oesophageal wall, which is frequently impossible with conventional radiology. A trial by HERTH et al. [20] showed that EBUS, in contrast to CT imaging, was highly reliable in differentiating external tumour invasion from impression of the tracheobronchial wall (94 versus 51\%). In total, 104 patients with central tumours were examined with EBUS and CT, and their tumours were classified as invasion or impression. All patients underwent surgery, and the findings were compared with the initial classification. In differentiating between airway infiltration and compression by the tumour, the US technique was superior to CT in sensitivity (89 versus $25 \%$ ) and specificity (100 versus $89 \%$ ). Thus, patients with supposed T4 tumours considered non-resectable by the radiologist could be operated on using a curative approach after EBUS.

\section{Peripheral lesions}

For histological diagnosis of peripheral intrapulmonary lesions by bronchoscopy, a fluoroscopic or CT guidance approach is the standard procedure. In a prospective study, it was shown that approaching those lesions through EBUS guidance yielded the same success rate $(\sim 75 \%)$ [21]. Recently, these data were confirmed by other groups, which also achieved a diagnostic yield of $75 \%$ using the miniprobe as a guidance tool for the forceps.

\section{Lymph node staging}

Under favourable conditions, even small lymph nodes ( $2-3 \mathrm{~mm}$ in size) can be detected by EBUS, and the internal structure (sinuses and folliculi), as well as small lymph vessels, can be analysed. The results of TBNA can be significantly improved (up to $85 \%$ ) by using endosonographic localisation of lymph nodes (figure 6) [22]. This improvement is especially evident for positions in which reliable endoscopic landmarks are missing, such as high and low paratracheal localisation.

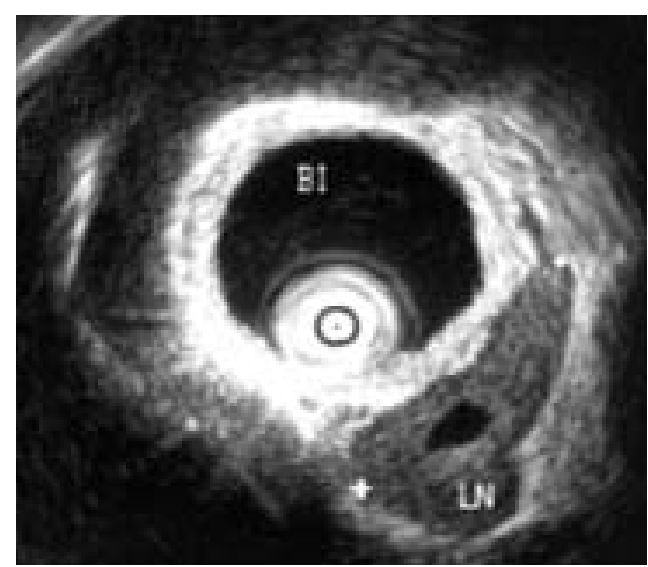

Figure 6

The EBUS miniprobe is located in the bronchus intermedius (BI). A lymph node (LN) is visible.

Lymph node staging is also the main indication for use of the new EBUS-TBNA scope.

EBUS-TBNA is performed by direct transducer contact with the wall of the trachea or bronchus. With this technique, a real-time puncture of the nodes is possible (figure 7).

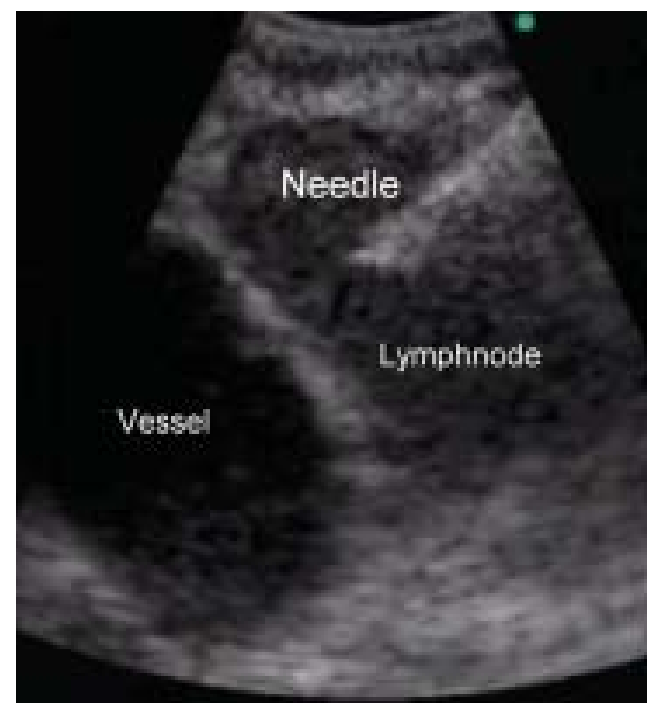

Figure 7

A real-time puncture of an enlarged lymph node with the new EBUS-TBNA scope. The tip of the needle can be seen in the node.

To date, five papers have been published on this procedure. KRASNIK et al. [16] reported on 11 patients in whom 15 lesions were punctured without complications. The lesions were located in the following regions: four in $10 \mathrm{~L}$, four in $10 \mathrm{R}$, one in $4 \mathrm{~L}$, three in $4 \mathrm{R}$, one in 1 , one in 7 , and one in $2 \mathrm{R}$. The lesions ranged $7-80 \mathrm{~mm}$ in size. Biopsies 
obtained through EBUS-FNA showed malignant cells in 13 lesions and benign cells in two.

In a second trial, YASUFUKU et al. [23] examined 70 patients with mediastinal $(n=58)$ and hilar lymph nodes $(n=12)$. The sensitivity, specificity and accuracy of EBUS-TBNA in distinguishing benign from malignant lymph nodes were 95.7, 100 and 97.1\%, respectively. There were no complications.

In a recent paper by RinToul et al. [24], EBUS-TBNA was used in 18 patients. Cytology revealed N2/N3 disease in 11 patients and provided a primary diagnosis in eight patients. Cytology results for EBUS-TBNA samples were negative in six patients, and mediastinoscopy or clinical follow-up confirmed this result in four. Sensitivity, specificity and accuracy for EBUS-TBNA were 85,100 and $89 \%$, respectively.

The largest trial reports the results of the technique in 502 patients [25]. A total of 572 lymph nodes were punctured, and 535 (94\%) resulted in a diagnosis. Biopsies were taken from all reachable lymph node stations $(2 \mathrm{~L}, 2 \mathrm{R}, 3,4 \mathrm{R}, 4 \mathrm{~L}, 7,10 \mathrm{R}$, $10 \mathrm{~L}, 11 \mathrm{R}$ and $11 \mathrm{~L}$ ). Mean \pm SD diameter of the nodes was $1.6 \pm 0.36 \mathrm{~cm}$ and the range was $0.8-3.2 \mathrm{~cm}$. The sensitivity was $92 \%$, specificity was $100 \%$ and the positive predictive value was $93 \%$. As with all other trials, no complications occurred.

\section{Conclusions}

The usefulness of US for chest physicians is firmly established. It is an easy-to-learn, low-cost method that extends the physician's diagnostic and interventional potential at the bedside in different lung and pleural diseases. Sonographic evidence of pleural nodules is a specific finding in patients with malignant effusions. Peripheral pulmonary consolidations are visible using US if extended up to the pleura. Pleuritis, pneumonia, lung cancers, pulmonary infarction and atelectasis can be imaged with regard to typical sonomorphology, but this has limited specificity. US-guided transthoracic puncture is minimally stressful for the patient, accurate, has a low rate of complications and is also cost-effective. Future research and developments should include formal training programmes for chest physicians to ensure a basic standard for chest US, and the aim to refine the application of chest ultrasonography for routine use for thoracentesis in the ICU and chest tube placement.
EBUS has been widely available for $>5$ years. A growing body of good-quality literature supports a significant role in airway assessment and procedure guidance. Its usefulness is especially well documented in lymph node staging via guided TBNA and in lending support for therapeutic decision making with regards to endoluminal or alternative treatment strategies for malignant airway abnormalities.

EBUS is a routine adjunct to endoscopy in many centres, and its role is expected to grow in the near future.

\section{Educational questions}

1. Which of the following statements regarding transthoracic ultrasound is correct?

a) It is a difficult and long-acting technique.

b) It helps to differentiate between pleural effusion and pleural thickening.

c) It can only be used in the ultrasound suite.

d) It requires local anaesthesia.

e) All answers are correct.

2. Which of the following statements regarding pneumothorax is correct?

a) It isn't visible by ultrasound.

b) With ultrasound, the amount is exactly calculable.

c) It needs a lot of experience.

d) It is visible with ultrasound.

e) All answers are correct.

3. Which of the following statements regarding EBUS is correct?

a) It is an easy technique.

b) It helps in therapeutic decisions in early cancer lesions.

c) It requires a special bronchoscope.

d) The procedure needs to be carried out under general anaesthesia.

e) All answers are correct.

4. Which of the following statements regarding EBUS-TBNA is correct?
a) It is an easy technique.
b) It helps in therapeutic decisions in early cancer lesions.
c) It requires a special bronchoscope.
d) The procedure needs to be carried out under general anaesthesia.
e) All answers are correct. 


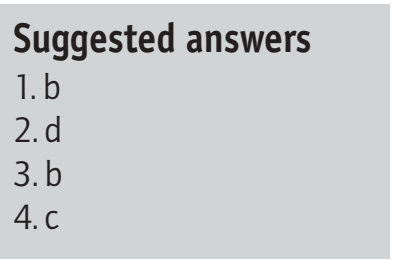

\section{Suggested further reading}

See references with comments in blue.

\section{References}

1. Mathis G. Thoraxsonography. I. Chest and pleura. Ultrasound Med Biol 1997; 23: 1131-1139.

2. Koh DM, Burke S, Davies N, Padley SPG. Transthoracic US of the chest: clinical uses and applications. Radiographics 2002; 22: 1 1. Perfect overview of transthoracic US.

3. Yang $P C$, Luh KT, Chang DB, Wu HD, Kuo SH. Value of sonography in determining the nature of pleural effusion: analysis of 320 cases. AJR Am J Roentgenol 1992; 159: 29-33.

4. Beck S, Bolcskei PL, Lessnau KD. Real-time chest ultrasonography: a comprehensive review for the pulmonologist. Chest 2002; 122: 1759-1773.

A topical overview.

5. Wu HD, Yang PC, Lee LN. Differentiation of lung abscess and empyema by ultrasonography. J Formos Med Assoc 1991; 90: 749-754.

6. Yu CJ, Yang PC, Wu HD, Chang DB, Kuo SH, Luh KT. Ultrasound study in unilateral hemithorax opacification: image comparison with CT. Am Rev Respir Dis 1993; 147: 430-433.

7. Gehmacher 0, Kopf A, Scheier M, Bitschnau R, Wertgen T, Mathis G. Can pleurisy be detected with ultrasound? Ultraschall Med 1999; 18: 214-219.

8. Lichtenstein D, Meziere G, Biderman P, Gepner A. The comet tail artifact: an ultrasound sign ruling out pneumothorax. Intensive Care Med 1999; 25: 383-388.

An interesting feasibility paper, especially for those working in intensive care.

9. Chung MJ, Goo JM, Im JG, Cho JM, Cho SB, Kim SJ. Value of high-resolution ultrasound in detecting a pneumothorax. Eur Radiol 2005; 15: 930-935.

10. Reissig A, Kroegel C. Accuracy of transthoracic sonography in excluding post-interventional pneumothorax and hydropneumothorax. Comparison to chest radiography. Eur J Radiol 2005; 53: 463-470.

11. Mathis G. Ultrasound diagnosis of pulmonary embolism. Eur J Ultrasound 1996; 3: 153-160.

12. Yang PC, Chang DB, Yu CJ, et al. Ultrasound guided core biopsy of thoracic tumors. Am Rev Respir Dis 1992; 146: 763-767.

13. Pan JF, Yang PC, Chang DB, Yu CJ, Kuo SH, Luh KT. Needle aspiration biopsy of malignant lung masses with necrotic centers: improved sensitivity with ultrasonic guidance. Chest 1993; 103: 1452-1456. State-of-the-art study on US-guided puncture.

14. Falcone F, Fois F, Grosso D. Endobronchial ultrasound. Respiration 2003; 70: 179-194. An up-to-date overview on EBUS.

15. Herth F, Becker HD. Endobronchial ultrasound of the airways and the mediastinum. Monaldi Arch Chest Dis 2000; 55: 36-45.

16. Krasnik M, Vilmann P, Larsen SS, Jacobsen GK. Preliminary experience with a new method of endoscopic transbronchial real time ultrasound guided biopsy for diagnosis of mediastinal and hilar lesions. Thorax 2003; 58: $1083-1086$. A recent study on EBUS-TBNA.

17. Kurimoto N, Murayama M, Yoshioka S, Nishisaka T, Inai K, Dohi K. Assessment of usefulness of endobronchial ultrasonography in determination of depth of tracheobronchial tumor invasion. Chest 1999; 115: 1500-1506.

18. Herth F, Becker HD. EBUS for early cancer detection. J Bronchol 2003; 10: 249-253.

19. Miyazu Y, Miyazawa T, Iwamoto Y, Kano K, Kurimoto N. The role of endoscopic techniques, laser-induced fluorescence endoscopy, and endobronchial ultrasonography in choice of appropriate therapy for bronchial cancer. J Bronchol 2001; 8: 10-16.

20. Herth FJ, Ernst A, Schulz M, Becker HD. Endobronchial ultrasound reliably differentiates between airway infiltration and compression by tumor. Chest 2003; 123: 458-462.

21. Herth F, Ernst A, Becker HD. Endobronchial ultrasound-guided transbronchial lung biopsy in solitary pulmonary nodules and peripheral lesions. Eur Respir J 2002; 20: 972-975. State-of-the-art study of EBUS-guided transbronchial lung biopsy.

22. Herth F, Becker HD, Ernst A. Conventional vs endobronchial ultrasound-guided transbronchial needle aspiration: a randomized trial. Chest 2004; 125: 322-325.

23. Yasufuku $K$, Chiyo M, Sekine $Y$, et al. Real-time endobronchial ultrasound-guided transbronchial needle aspiration of mediastinal and hilar lymph nodes. Chest 2004; 126: 122-128.

24. Rintoul RC, Skwarski KM, Murchison JT, Wallace WA, Walker WS, Penman ID. Endobronchial and endoscopic ultrasoundguided real-time fine-needle aspiration for mediastinal staging. Eur Respir J 2005; 25: 416-421.

25. Herth FJF, Eberhardt R, Vilmann P, Krasnik M, Ernst A. Real-time, endobronchial ultrasound-guided transbronchial needle aspiration: a new method for sampling mediastinal lymph nodes. Am J Respir Crit Care Med 2005; 49: 157. 